\title{
The Effects of Mindfulness Meditation on Emotion Regulation, Cognition and Social Skills
}

\author{
Thomas M. Jones \\ The Paradox Process, New York, NY, USA
}

Doi: 10.19044/esj.2018.v14n14p18 URL:http://dx.doi.org/10.19044/esj.2018.v14n14p18

\begin{abstract}
This review seeks to bridge the gap between the separate but interacting mechanisms of emotion regulation and cognition, as well as their potential relationship with mindfulness meditation tools. By way of mindfulness meditation, individuals can learn how to regulate their emotions in a way that aversive stimuli will be viewed objectively; thus, the person can be free of attachment from said negative feelings. Knowing this, there is a potential link between emotion regulation processes and cognitive mechanisms that allow such regulation to take place, including selective or focused attention and inhibition. The literature on this theory so far has been inconsistent, however more claims suggest that there is a relationship between the two. This review initially speaks to existing mindfulness research and its implications on emotion regulation and cognitive processes. We then discuss emotion and the underlying processes and potential benefits of emotion regulation practice, as they are related to mindfulness mechanisms. Cognition, and the relationship between emotional intelligence and social skills are also discussed. Finally, we put it all together by suggesting a proactive mindfulness technique, which proves to be beneficial for each area mentioned.
\end{abstract}

Keywords: Mindfulness, Emotional Intelligence, Cognition, Social Skills, Self-regulation

\section{Mindfulness Research}

Mindfulness originated in the Eastern Buddhist traditions and has become increasingly popular in Western psychological practice. It has been indicated to improve overall mental health and general wellbeing. Mindfulness teaches one to focus on the present moment, as well as to become aware of and accept one's feelings and emotions. According to Kang and Whittingham (2010), mindfulness is a tool that contributes to one's journey to enlightenment. They argue that proper ethical and moral judgments are made based on mindfulness techniques, and ultimately help to bring forth wisdom, 
knowledge, and genuine happiness (Kang \& Whittingham, 2010). Selfawareness and knowledge of one's internal emotions and feelings allows him or her to utilize mindfulness tools. Coffey, Hartman, and Fredrickson (2010) compare Western and Buddhist psychology and state that both schools of thought teach individuals how to free themselves from their feelings in order to escape their depressive states.

Dialectical Behavioral Therapy skills training covers four main categories that are pertinent to this review: mindfulness, emotion regulation, interpersonal effectiveness, and distress tolerance (Linehan, 1993, 2015). Patients are trained to use mindfulness in daily activities which aims to reduce patients' difficulties in regulating emotions: impulse control, interpersonal relationships, and self-image (Linehan, 2015). As described by Linehan (2015), DBT $®$ Mindfulness skills training is essential to managing the moment, with the emphasis on developing the appropriate skills to manage the situation of the moment.

Jon Kabat-Zinn, a pioneer in mindfulness intervention research, along with colleagues observed a sample of psoriasis patients, who reported alleviated or decreased psoriasis symptoms after being exposed to a Mindfulness-Based Stress Reduction (MBSR) program compared to a control group (Kabat-Zinn et al., 1998). This work not only uncovered the physical benefits of mindfulness intervention, but also set forth explorations on the effects of stress as well. Similarly, Davidson et al. (2003) introduced an 8week mindfulness-based stress reduction program to healthy employees in their work environment. As expected, the mindfulness intervention changed the brain functions of the participants in specific ways, thus reducing stress symptoms and increasing their sense of wellbeing (Davidson et al., 2003).

There have been some difficulties in understanding the construct of mindfulness. Typically, in Western psychology, definitions of mindfulness focus on the concentration aspect of this practice, according to Grabovac, Lau, and Willett (2011). They proposed a "Buddhist Psychological Model", which provides several mechanisms such as attention regulation, insight, acceptance/compassion, etc. These functions help to adaptively regulate subjective experience. In addition, Grabovac et al., (2011) also relate this model to mindfulness practice, as the model itself is considered a "model of the underlying mechanisms of mindfulness," (p.161).

One operational definition of mindfulness comes from Bishop et al., (2004), who argue that mindfulness includes components comprised of both attention and awareness; Linehan (1993) mentions that mindfulness requires full participation in the experience; Brown and Ryan (2003) note that this process requires one to attend to the present moment (as cited in Coffey et al., 2010, p. 236). Previous researchers have attempted to find an accurate measure of mindfulness, which took nearly a decade before they could come to a 
consensus regarding the underlying components of mindfulness that could be empirically observed (Coffey et al., 2010). Moore and Malinowski (2009) suggest that two main perspectives on the processes of mindfulness meditation - one that focuses on the MBSR intervention program being used in correspondence to medical settings; the other perspective is one that chooses to focus on the cognitive, emotional, and neurophysiological changes that result from prolonged practice.

\section{Emotion Regulation}

Individuals tend to associate an emotionally charged feeling with a seemingly automatic response and, as a result, accept what they are feeling as reality (Garland, Gaylord, \& Park, 2009). However, these feelings, like any other behavior, are learned, and thus can be unlearned. Possessing tools to cope with stress and problem solve in a healthy and productive manner can combat the potential negative social-emotional and psychological effects that stress can have on adults and children alike. Stress management styles vary across individuals and may serve as determinants of future depressive episodes. One of the most prominent styles of emotion regulation is cognitive reappraisal, which works as a protective factor when dealing with particularly stressful situations and may serve as a thin line between stress and depression (Troy, Wilhelm, Shallcross, \& Mauss, 2010). This concept of positive reappraisal is one that allows an individual to reevaluate their initial reactions of whatever stimulus they have experienced and adapt to it in a way that promotes positivity and self-actualization.

The ongoing efforts of researchers have emphasized the way that humans become aware of their emotions and accept them objectively. The relationship between mindfulness and emotion regulation is one that continues to grow stronger, as new evidence emerges across studies. A growing body of research supports the claim that emotion is a key target of mindfulness practice, and therefore learning to regulate one's emotions will have beneficial effects to their well-being. For example, a greater tendency to attend to one's present moment experience and the acceptance of it were associated with a greater ability to identify and manage one's negative emotions (Coffey et al., 2010). This means that awareness of a negative emotional state can be controlled once an individual is made aware of it, and thus can be reversed. Practicing this awareness can help reverse the effects of ruminative thinking and mood swings, which are said to be causes of depression.

It is important to make the distinction here that mindfulness training should focus on reappraising secondary emotions, whereas primary emotions are more of an automatic process, and secondary emotions are mere responses to those initial feelings after being exposed to an affective stimulus (Coffey et al., 2010). According to Troy et al., (2010), cognitive reappraisal aims to alter 
individuals' perspectives of their appraisals rather than the stressful event they have been exposed to. In their study to determine if cognitive reappraisal ability served as a protective factor when measuring in correspondence with "high life stress," Troy et al., (2010) measured down regulations in participants after watching emotion-evoking clips along with self-reports of emotion regulation and skin conductance levels. They found that although group differences were not significant, participants who were attempting to correctly use cognitive reappraisal strategies were showing a shift in sadness levels (Troy et al., 2010). Cognitive reappraisal and mindfulness tend to go hand in hand when dealing with these introspections. Although training in mindfulness strategies proves to be effective in turning negative emotions into positive ones, Garland et al., (2010) note that these mechanisms are more of a "mode" than a "trait," and are best sustained when the individual is consistent with training this form of attention. Enduring change is likely when one learns to make these "modes" a more automatic function of regulation capacity (Greenberg, 2008).

Until recently, emotions were viewed as "distractions... something to be controlled or avoided," as mentioned by Beck (1976) (as cited in Greenberg, 2008). However, because emotions are now considered to be another mechanism of information processing (Greenberg, 2008), they are vital to our knowledge and how we move about in the world. Emotions work as affective resources that individuals use to make sense of specific life events they come across. Studies show that emotion is adaptive in that it performs as a rapid-action reflex in order to focus people on the relevance of an event that occurs. Our emotional systems tend to sort and prioritize those life events according to their significance, or relevance, to our sense of self. Overregulation, or avoidance, of one's emotions can prove to be detrimental to one's mental and physiological health (Greenberg, 2008). However, appropriate awareness and corresponding display of emotion help to develop proactive coping styles, which are necessary for maintaining emotional balance. However, in more recent studies, researchers are starting to shift their focus on the interaction between emotion and cognition. Although this particularly novel area of study has certainly become a topic of interest, the literature on this subject remains inconsistent. Fortunately, there is growing evidence to support the notion that emotion regulation and cognitive flexibility are increasingly related. Specifically, research suggests that these two concepts are "separate but interacting mental functions," (Greenberg, 2008).

\section{Relationship between Emotion and Cognition}

Studies have shown that neutral, positive, or negative stimuli have had an effect on attention orientation as well as emotional interference in the presence of a cognitive task. Moore and Malinowski (2009) found that there 
was a positive effect in experienced meditators while performing the Stroop task. Furthermore, brief 4-day mindfulness meditation training has shown to have similar effects on cognition. Zeidan, Johnson, Diamond, David, \& Goolkasian, (2010) found that a brief mindfulness meditation intervention improved performance on several cognitive tasks; however, there was no effect on mood scales which were also utilized. Blair et al., (2007) found that individuals had increased reaction times, due to distraction, by both positive and negative emotional stimuli when performing the Stroop task. On the contrast, Ortner, Kilner, \& Zelazo (2007) employed two studies; the first one, a correlational approach, found that mindfulness meditation experience was correlated to reduced emotional interference on a cognitive task. It is worth mentioning that in the second study, participants still oriented attention toward unpleasant stimuli in the emotional interference task, however those who practiced mindfulness meditation also showed increased rapid disengagement from the unsolicited stimuli, as well as a decrease in emotional interference. The limitation of these two studies, however, is that they only focus on the influence of emotion on attention and fail to observe other cognitive mechanisms such as memory, inhibition, or learning.

When discussing the relationship between emotion and cognition, it is important to address the fact that the same brain regions are activated when being aroused emotionally or cognitively. The amygdala is one of the main systems in the human brain that is in control of emotional processes and is also known to detect affectively arousing stimuli (Scherpiet, Holtforth, Herwig, \& Bruhl, 2014). Phelps (2006) notes that the anatomy of the amygdala is what makes it an ideal location for influencing emotional response systems. Additionally, the prefrontal cortex is the region of the brain most associated with cognition and executive functions - mainly selective attention, working memory, inhibition, and monitoring. Mindfulness and emotion regulation techniques manipulate this area, and as a result, manipulate the underlying mechanisms that make up our cognition (Holzel et al., 2011).

Mindfulness meditation in itself calls for constant vigilance on a moment-to-moment basis. Being that the act of being "mindful" is focusing on the present moment, this alone calls on top-down processes such as selective attention; bottom-down processes, or the more automatized processes of the brain, are exactly what mindfulness techniques work on. More specifically, attention and awareness have been found to be manipulated by mindfulness practice. While this traditional practice calls for emotion regulation, it also has some promising effects on cognitive performance. Teper, Segal, and Inzlicht, (2013) describe the relationship between mindfulness, executive control, and emotion regulation, as interchangeable mindfulness improves executive control, which then helps to improve attention and awareness. 


\section{Emotion Regulation and Social Competence}

There has been much debate about whether the ability to regulate one's emotions has a negative effect on the ability to feel empathic towards others. In one study, Lebowitz and Dovidio (2015) describe how emotion regulation is said to "facilitate the collapse of compassion", specifically, collapse of compassion towards groups. Lebowitz and Dovidio (2015) wanted to find whether emotion regulation was related to negative social attitudes and avoidance of altruistic behaviors. Results showed that in both studies, those who regulated their emotions showed a decrease in helping behaviors. Although this study found such negative association, they emphasized that the emotion regulation process that was implemented on the subjects was one that involved suppressing aversive emotions, which is not necessarily a beneficial technique, considering that suppression of emotions does not necessarily equate to healthy regulation. In fact, it is well known that suppression is positively linked to increased depressive symptoms (Troy et al., 2010).

Both ability and awareness of emotion regulation prove to be of importance in order to engage in prosocial behavior. Mauss et al., (2011) mention that positive emotions are only beneficial to the effort of social connectedness when they are experienced genuinely; that is, when an individual accurately displays his or her emotions in a way that corresponds to the way they are feeling internally. In order to communicate and form healthy bonds with others, people should engage in such behavior that encourages genuine displays of affection and compassion; otherwise, the inconsistency will disrupt the relationship they are trying to sustain. This "accuracy," as termed by Mauss et al., (2011), can only be attained by awareness and acceptance of one's emotions, which is a particular function that is used in emotion regulation techniques such as mindfulness meditation (Holzel et al., 2011).

Anxiety and an inability to cope with stress-provoking stimuli are inevitable elements in the lives of most youth in our society. Approximately 1 in 5 adolescents in the general population are susceptible to mental disorders that have long-term effects; $11.2 \%$ of those being mood disorders, $8.3 \%$ being anxiety disorders, and $9.6 \%$ behavior disorders ("Youth Topics/Mental Health," 2010). Behavior and social-emotional challenges negatively impact academic achievement, relationships with peers and adults, social development, and stresses teachers and the communities at large (Walker, Ramsey, \& Gresham, 2004). Building resilience in children and adolescents has shown to help counteract the effects of at-risk behaviors and promote emotional, social, and academic well-being (Dishion \& Connell, 2006).

While there is extensive research on emotion regulation strategies, the majority of the research only focuses on college undergraduate students, with this being the most accessible population. The demand for learning such 
strategies, however, should not solely be intended for college students alone. Children and adolescents benefit most from learning positive emotion regulation, as they are exposed at an early age and thus make their practice more sustainable (Ames, Richardson, Payne, Smith, \& Leigh, 2014). Growing research elucidates the many positive and long-lasting benefits of mindfulness, particularly with the adult population. Translating these skills to children and adolescents via mindfulness tools and techniques can facilitate greater resiliency and psychological benefits from earlier, more formative years, which studies show can predict the likelihood of positive future outcomes and characteristics associated with overall life satisfaction (Ames et al., 2014).

Social behavior among adolescents is a particularly important interest to researchers, because of the everyday problems that this age group experiences. Specifically, high school students are more prone to experience negative emotions that may prove to be detrimental in the long-term. They are exposed to a variety of factors that may lead to low academic performance in school such as bullying, social constructs of popularity, and self-image. While there have been previous studies on the effects of mindfulness on social behavior of primary and secondary school students, it is important to realize that some of these studies have a tendency to focus more on school performance and cognitive abilities such as attention and concentration (Ricarte, Ros, Latorre, \& Beltran, 2015), rather than just self-esteem and social connections. In another mindfulness study, Franco, Mañas, Cangas, and Gallego (2010) sought to examine the effectiveness of a meditation program on students' academic performance, self-concept, and reduction of anxiety levels in high school students. Their results showed that after the implementation of the program, academic performance and self-concept in the experimental group improved significantly while simultaneously reducing their anxiety levels.

Social status among peers has been noted to be a predictor of internalized disorders (Merrell \& Gimpel, 1998). There have been studies that search for a link between emotional intelligence and social behavior. It is interesting to note that while this information is particularly vital for the adolescent population, there are very few studies focusing on the trait emotional intelligence of individuals in this particular age group (Mavroveli, Petrides, Rieffe, \& Bakker, 2007). Trait emotional self-efficacy is related to social skills, and thus, adolescents with high self-efficacy are more likely to react to certain social situations with appropriate behavior, thus engaging in healthy peer relationships and improving their sense of self and overall psychological well-being (Mavroveli et al., 2007).

In a sample of Dutch adolescents, Mavroveli et al., (2007) found that students considered those peers who scored higher on emotional intelligence more cooperative. Trait emotional intelligence is associated with prosocial 
behavior and social competence, particularly important because knowing how to regulate one's emotions acts as a reference for determining the feelings and emotions of others. In another study, student participants in a mindfulness education program reported significant increases in optimism and improvements on classroom social behaviors as reported by their teachers (Schonert-Reichl \& Lawlor, 2010). Social skills serve as an internal awareness that can be learned through mindfulness and have also been suggested to be an important precondition for empathic responses. The implementation of a Mindfulness-Based Stress Reduction program found that socially anxious patients showed quicker decreases in amygdala activation (Goldin \& Gross, 2010). In addition, there are Neuroscientific findings that the same brain regions are activated during tasks of both interoceptive awareness and social cognition (Holzel et al., 2011).

\section{Proactive vs. Reactive Efforts}

One complication that arises with the therapeutic efforts to increase emotion regulation ability is that the distinction between the ability to selfregulate and the action of self-regulation remains ambiguous. Additionally, emotion is often interpreted as those feelings that one reacts to, instead of being interpreted as a process that works to provide meaning to associated life events in order to make them meaningful. Specific emotions are associated with specific memories. The best way to dissociate the two is to change the way a person views the event, which eventually changes the emotion connected to it (Greenberg, 2008). However, this method is reactive, in that it focuses on changing a certain connection that is already implanted in our brains. Rather, the effects of emotion regulation are more sustainable if a proactive method of dissociation is applied. Teper et al., (2013) note that mindfulness is "antecedent-focused." Having a proactive mind state creates a schema in our mental framework that will rewire automatic processes to engage in our emotional experiences objectively, rather than believing that they are a part of us. This passing view of emotional events is paramount in reevaluating significance of emotional stimuli and realizing that they are not part of us, thus making it easier to remove our attachment from our emotions and regulating them effectively.

Ruminative thinking is the tendency to constantly think about the different components of an upsetting situation. While this way of thinking can make one aware of his or her feelings, it is actually harmful to one's mental well-being. There are a number of studies that discuss how mindfulness-based intervention helps to conquer rumination and presents introspective solutions that allow an individual to accept themselves and their surroundings in the present moment. Coffey et al., (2010) explain how focusing on the present prevents one from succumbing to his or her ruminative thoughts. Heeren and 
Philippot (2010) reported significant rumination symptom reduction after a mindfulness intervention. They argue that maladaptive rumination has the tendency to "reduce concreteness of thinking," whereas complex cognitive processes equate to a higher probability to fixate on intrusive thoughts when confronted with emotional difficulties. In contrast, adaptive rumination holds concrete representations of stimuli and promotes low-level information processing, which is associated with the underlying mechanisms of mindfulness as low-level processing allows you to focus on present- moment feelings and sensations (Heeren \& Philippot, 2010). Forster et al., (2006) support this view by stating rumination is encouraged by focused attention processes, whereas positive emotions are associated with distributed attention (as cited in Raffone, Tagini, and Srinivasan, 2010). Of course, this view does not go unchallenged, as Holzel et al., (2011) argue that focused attention on internal events is necessary in order to gain bodily awareness and avoid distracting stimuli. Despite these contrasting views, both focused and distributed attentions are necessary in order for continual awareness to take place.

The downfall of taking reactive action when dealing with ruminative thinking is that successive negative thoughts already do a great deal in hindering regulation processes, thus making the effect of any intervention harder to sustain. This specific attention on ruminative thoughts inhibits individuals from focusing on the act of letting go, as they do not even realize what they are doing. Thus, it is important to implement intervention programs that teach individuals to consciously recognize negative stimuli before they appear in the mental framework, as it is an essential technique that proves to be more beneficial than any reactive effort. From a neurological standpoint, it is possible to train structural regions so that those that target reflection and emotional experience will strengthen these connections, thus growing this circuitry will support healthy regulation patterns across the lifespan (Zelazo \& Lyons, 2012).

One example of promoting proactive efforts among individuals is teaching resilience. For example, Zimmerman, et al., (2013) propose an intervention program for at-risk youth in order to enhance positive development. They also describe how resilience theory is a "strength-based model," as it focuses on enhancing promotive factors instead of on reducing risk exposure. It is important to implement these teachings to younger age groups so that the effects will last long and thus build resilience, healthy risktaking, and self-actualization. Dishion and Connell (2006) refer to "effortful attentional control" (pp. 126-127), which is an emerging process that seeks to find common ground among self-regulatory processing ability in specific contexts among children and adolescents. Learning self-regulation as well as 
resilience strategies are beneficial for youth age groups in various social as well as educational situations.

\section{Conclusion}

In summary, mindfulness meditation is a growing practice in Western psychology, although there is still much to be explored. Several studies have proved that the implementation of mindfulness interventions on adolescents and adults' results in adaptive coping, resilience, and self-regulation. The underlying mechanisms of mindfulness, including attention, awareness, and acceptance of emotional stimuli, all serve to help an individual gain a detached perspective of their emotional experiences, thus promoting healthy coping strategies that lead to better psychological health and well-being.

Emotions are given meaning and importance according to our personal experiences, resulting in maladaptive attachments and false representations that they are a part of the self, a burden we feel we must hold onto. The powerful effect of practicing mindfulness, or more general emotion regulation strategies, is that one learns that emotions are simply transient events that constantly occur and disappear, thus, making it easier to view them objectively and keep them in check. One of the most effective regulation strategies is cognitive reappraisal, which allows for a "second look", a more objective view of emotional experiences in order to change the way that they are interpreted. Cognitive reappraisal has been well known to reverse the effects of stress and has important implications for reversing depressive symptoms (Coffey et al., 2010). Due to these effects, it is becoming more apparent across studies that emotion regulation proves to be beneficial for overall mental health.

Executive functions such as inhibition, selective attention (or setshifting), and working memory have all been tested in regard to levels of mindfulness meditation or emotion regulation experience. Several studies have in fact found that cognitive reappraisal ability, as well as mindfulness meditation interventions, have had positive effects on executive functioning (Gallant, 2016; Heeren et al., 2009). In addition, neurological studies have shown that emotional and cognitive functions are activated by the same regions in the brain (Holzel et al., 2011), such as the amygdala (Greenberg, 2008; Phelps, 2006) and the prefrontal cortex (Scherpiet et al., 2014). Knowing this, the potential link between emotion regulation processes and cognitive mechanisms that allow such regulation to take place continues to grow stronger.

Although the relationship between emotion and cognition has not been set in stone, it is tempting to accept that the two functions are interdependent. However, there are inconsistencies in various studies that sought to show the effects of emotion regulation on cognitive tasks, such as the Stroop task (Moore \& Malinowski, 2009; Zeidan et al., 2010) and the Hayling task 
(Heeren et al., 2009). While most of these experiments did find significant main effects and interactions between these two functions, it is vital for theoretical sake to remember that the focus was concentrated more so on just one specific domain of cognitive functioning - inhibition (Gallant, 2016; Moore \& Malinowski, 2010). Gallant (2016) notes that current literature has yet to determine whether specific aspects of executive functioning can be improved, or if they can only be improved as a whole. Indeed, inhibitory ability overlaps with mindfulness strategy, as this cognitive control helps to avoid distractions and focus on the present moment. However, in order to make the relationship between cognition and emotion regulation strategies clearer, it would be more appropriate for researchers to try to establish that there are definitive links to other cognitive functions such as working memory, learning ability, and conflict monitoring.

Emotion regulation ability has direct positive effects on mental wellbeing and can regulate stress and depressive symptoms (Coffey et al., 2010). Regarding the importance of social competence, Mavroveli et al., (2007) found that trait emotional self-efficacy is in fact related to social skills, and social status is a predictor of internalized disorders (Merrell \& Gimpel, 1998). Studies on the benefits of emotion regulation on social competence have been fairly consistent; emotional intelligence can be directly linked to prosocial behavior (Dishion \& Connell, 2006; Mavroveli et al., 2007). As a result, emotional intelligence practice can be implemented in institutions and settings where most social interactions take place - schools, workplaces, etc. One particular finding revealed that learning mindfulness techniques improved classroom attention in adolescents (Ricarte et al., 2015). More importantly, adolescents benefit the most from learning such strategies, as they are exposed to social situations that can deter their sense of self and intensify emotional disorders. Although the statistics of anxiety disorders in adolescents is alarming, there are very limited studies on the effects of social competence on adolescents. A few studies have found positive effects of mindfulness meditation and emotion regulation strategies on social behavior (Mavroveli et al., 2009; Schonert-Reichl \& Lawlor, 2010) and improvements in self-concept (Franco et al., 2010). This can be explained by the fact that emotional awareness allows one to understand his or her own emotions, and in turn they are able to accurately appraise the emotions of others. Thus, they'd have the proper knowledge to react to others' emotions appropriately.

There are contrasting views on whether the effects of emotion regulation are more sustainable if a person changes their actual appraisals rather than their feelings themselves. However, these debates fail to mention a third solution, which implies that an "antecedent-focused model" (Teper et al., 2013) will help a person to identify the feelings in which they are about to experience, in a way that decreases any attachment to those feelings, so that 
reasonable behavioral action is not compromised. This "meta-awareness" of emotion is proactively based in the idea that non-attachment decreases the significance that these aversions hold, and thus, helps to decrease maladaptive ruminative thinking. Altogether, mindfulness meditation is a technique that teaches individuals emotion regulatory practices such as cognitive reappraisal, which ultimately is beneficial to overall psychological well-being and thus promoting a healthy, well-balanced life.

\section{References:}

1. Ames, C.S., Richardson, J., Payne, S., Smith, P., \& Leigh, E., (2014). Innovations in Practice: Mindfulness-based Cognitive Therapy for Depression in Adolescents. Child and Adolescent Mental Health, 19, 74-78.

2. Blair, K.S., Smith, B.W., Mitchell, D.G.V., Morton, J., Vythilingam, M., Pessoa, L., ... Blair, R.J.R, (2007). Modulation of Emotion by Cognition and Cognition by Emotion. NeuroImage, 35, 430-440.

3. Brown, K.W. \& Ryan, R.M., (2003). The Benefits of Being Present: Mindfulness and Its Role in Psychological Well-Being. Journal of Personality and Social Psychology, 84, 822-848.

4. Coffey, K.A., Hartman, M., \& Fredrickson, B.L., (2010). Deconstructing Mindfulness and Constructing Mental Health: Understanding Mindfulness and its Mechanisms of Action. Mindfulness, 1, 235-253.

5. Compas, B.E., Jaser, S.S., Dunbar, J.P., Watson, K.H., Bettis, A.H., Gruhn, M.A., \& Williams, E.K., (2014). Coping and Emotion Regulation from Childhood to Early Adulthood: Points of Convergence and Divergence. Australian Journal of Psychology, 66, 71-81.

6. Davidson, R.J., Kabat-Zinn, J., Schumacher, J., Rosenkranz, M., Muller, D., Santorelli, S.F., ... Sheridan, S.F., (2003). Alterations in Brain and Immune Function Produced by Mindfulness Meditation. Psychosomatic Medicine, 65, 564-570.

7. Dishion, TJ. \& Connell, A., (2006). Adolescents' Resilience as a SelfRegulatory Process: Promising Themes for Linking Intervention with Developmental Science. Annals of the New York Academy of Sciences, 1094, 125-138.

8. Franco, C., Mañas, I., Cangas, A.J., \& Gallego, J., (2010). The Applications of Mindfulness with Students of Secondary School: Results on the Academic Performance, Self-Concept and Anxiety. Knowledge Management, Information Systems, E-Learning, and Sustainability Research, 111, 83-97. 
9. Gallant, S., (2016). Mindfulness Meditation Practice and Executive Functioning: Breaking Down the Benefit. Consciousness and Cognition, 40, 116-130.

10. Garland, E., Gaylord, S., \& Park, J., (2009). The Role of Mindfulness in Positive Reappraisal. Explore, 5, 37-44.

11. Goldin, P.R. \& Gross, J.J., (2010). Effects of Mindfulness- Based Stress Reduction (MBSR) on Emotion Regulation in Social Anxiety Disorder. Emotion, 10, 83-91.

12. Grabovac, A.D., Lau, M.A., \& Willett, B.R., (2011). Mechanisms of Mindfulness: A Buddhist Psychological Model. Mindfulness, 2, 154166.

13. Greenberg, L., (2008). Emotion and Cognition in Psychotherapy: Transforming Power of Affect. Canadian Psychology, 49, 49-59.

14. Heeren, A. \& Philippot, P., (2011). Changes in Ruminative Thinking Mediate the Clinical Benefits of Mindfulness: Preliminary Findings. Mindfulness, 2, 8-13.

15. Holzel, B.K., Lazar, S.W., Gard, T., Schuman-Olivier, Z., Vago, D.R., \& Ott, U., (2011). How Does Mindfulness Meditation Work? Proposing Mechanisms of Action From a Conceptual and Neural Perspective. Perspectives on Psychological Science, 6, 537-559.

16. Kabat-Zinn, J., Wheeler, E., Light, T., Skillings, A., Scharf, M.J., Cropley, T.G., ... Bernhard, J.D., (1998). Influence of a Mindfulness Meditation-Based Stress Reduction Intervention on Rates of Skin Clearing in Patients With Moderate to Severe Psoriasis Undergoing Phototherapy (UVB) \& Photochemotherapy (PUVA). Psychosomatic Medicine, 60, 625-632.

17. Kang, C. \& Whittingham, K., (2010). Mindfulness: A Dialogue between Buddhism and Clinical Psychology. Mindfulness, 1, 161-173.

18. Lebowitz, M.S. \& Dovidio, J.F., (2015). Implications of Emotion Regulation Strategies for Empathic Concern, Social Attitudes, and Helping Behavior. Emotion, 15, 187-194.

19. Linehan, M. (2015) Cognitive-Behavioral Treatment of Borderline Personality Disorder, JAMA Psychiatry. (2015 May;72)(5):475-82. doi: 10.1001/jamapsychiatry.2014.3039., Dialectical behavior therapy for high suicide risk in individuals with borderline personality disorder: a randomized clinical trial and component analysis.

20. Mauss, I.B., Shallcross, A.J., Troy, A.S., John, O.P., Ferrer, E., Wilhelm, F.H., \& Gross, J.J., (2011). Don't Hide Your Happiness! Positive Emotion Dissociation, Social Connectedness, and Psychological Functioning. Journal of Personality and Social Psychology, 100, 738-748. 
21. Mavroveli, S., Petrides, K.V., Rieffe, C., \& Bakker, F., (2007). Trait Emotional Intelligence, Psychological Well-Being and Peer-Rated Social Competence in Adolescence. British Journal of Developmental Psychology, 25, 263-275.

22. Merrell, K., \& Gimpel, G. (1998). Social Skills of Children and Adolescents: Conceptualization, Assessment, Treatment. Mahwah, NJ: Lawrence Erlbaum Associates.

23. Moore, A. \& Malinowski, P., (2009). Meditation, Mindfulness and Cognitive Flexibility. Consciousness and Cognition, 18, 176-186.

24. Ortner, C.N.M., Kilner, S.J., \& Zelazo, P.D., (2007). Mindfulness Meditation and Reduced Emotional Interference on a Cognitive Task. Motivation and Emotion, 31, 271-283.

25. Phelps, E.A., (2006). Emotion and Cognition: Insights from Studies of the Human Amygdala. Annual Review of Psychology, 57, 27-53.

26. Raffone, A., Tagini, A., \& Srinivasan, N., (2010). Mindfulness and the Cognitive Neuroscience of Attention and Awareness. Zygon, 45, 627646.

27. Ricarte, J.J., Ros, L., Latorre, J.M., \& Beltran, M.T., (2015). Mindfulness-Based Intervention in a Rural Primary School: Effects on Attention, Concentration, and Mood. International Journal of Cognitive Therapy, 8, 1-11.

28. Schonert-Reichl, K. \& Lawlor, M.S., (2010). The Effects of a Mindfulness-Based Education Program on Pre- and Early Adolescents' Well-Being and Social and Emotional Competence. Mindfulness, 1, 137-151.

29. Teper, R., Segal, Z.V., \& Inzlicht, M., (2013). Inside the Mindful Mind: How Mindfulness Enhances Emotion Regulation Through Improvements in Executive Control. Current Directions in Psychological Science, 22, 449-454.

30. Troy, A.S., Shallcross, A.J., Davis, T.S., \& Mauss, I.B., (2013). History of Mindfulness-Based Cognitive Therapy is Associated with Increased Cognitive Reappraisal Ability. Mindfulness (NY), 4, 213222.

31. Troy, A.S., Wilhelm, F.H., Shallcross, A.J., \& Mauss, I.B., (2010). Seeing the Silver Lining: Cognitive Reappraisal Ability Moderates the Relationship Between Stress and Depressive Symptoms. Emotion, 10, 783-795.

32. Walker, H. M., Ramsey, E. \& Gresham, F. M. (2004). Antisocial behavior in school: Evidence-based practices (2nd ed.). Belmont, CA: Thomson/Wadsworth. 
33. Youth Topics, Mental Health Prevalence. (2010). Retrieved from https://youth.gov/youth-topics/youth- mental-health/prevalancemental-health-disorders-among-youth

34. Zeidan, F., Johnson, S.K., Diamond, B.J., David, Z., \& Goolkasian, P., (2010). Mindfulness Meditation Improves Cognition: Evidence of Brief Mental Training. Consciousness and Cognition, 19, 597-605.

35. Zelazo, P.D. \& Lyons, K.E., (2012). The Potential Benefits of Mindfulness Training in Early Childhood: A Developmental Social Cognitive Neuroscience Perspective. Child Development Perspectives, $6,154-160$.

36. Zimmerman, M.A., Stoddard, S.A., Eisman, A.B., Caldwell, C.H., Aiyer, M., \& Miller, A., (2013). Adolescent Resilience: Promotive Factors That Inform Prevention. Child Development Perspectives, 7, 215-220.

37. Zylowska, L., Ackerman, D.L., Yang, M.H., Futrell, J.L., Horton, N.L., Hale, T.S. ... Smalley, S.L., (2007). Mindfulness Meditation Training in Adults and Adolescents with ADHD. Journal of Attention Disorders, 11, 737-746. 